\title{
The expression of syndecan-1 in psoriatic epidermis
}

\author{
Davor Tomas · Majda Vučić • Mirna Šitum • \\ Božo Krušlin
}

Received: 10 January 2008 / Revised: 19 February 2008 / Accepted: 11 March 2008 / Published online: 2 April 2008

(C) The Author(s) 2008

\begin{abstract}
Psoriasis is a chronic inflammatory skin disease characterized by exaggerated keratinocyte proliferation. Current opinion indicates that psoriasis is driven by $\mathrm{T}$ cellmediated immune responses targeting keratinocytes. However, psoriasis cannot be explained solely on the basis of Tcell activation, and it is likely that an intrinsic alteration in epidermal keratinocytes plays a very important role in disease expression. Syndecans comprise a major family of cell surface heparan sulfate proteoglycans. Several studies indicate their role in adhesion, cell-extracellular matrix interactions, migration, keratinocyte proliferation and differentiation, inflammation, and wound healing. To determine the expression of syndecan-1 in psoriasis, skin samples from 29 patients with fully developed psoriasis and skin samples from 14 healthy volunteer persons with no personal or family history of psoriasis were immunohistochemically examined using monoclonal antibody against syndecan- 1 . The expression of syndecan- 1 was analyzed in whole mount section of psoriatic and non-psoriatic skin biopsies under high magnification $(400 \times)$. In addition, the intensity and topography of reaction in the cell, as well as localization of positive cells in the epidermis were evaluated. Strong syndecan-1 reactivity in epidermal cells in all
\end{abstract}

D. Tomas $(\square) \cdot$ M. Vučić $\cdot$ B. Krušlin Ljudevit Jurak Department of Pathology, Sestre Milosrdnice University Hospital,

Vinogradska 29, 10000 Zagreb, Croatia

e-mail: dtomas@kbsm.hr

M. Šitum

Department of Dermatovenerology, Sestre Milosrdnice University Hospital,

Vinogradska 29, 10000 Zagreb, Croatia non-psoriatic and psoriatic samples was observed. Statistical analysis showed no significant differences between two analyzed groups $(P>0.05)$. In normal skin syndecan-1 was expressed in full thickness of the epidermis. The strongest reaction was observed in membranes and intercellular junctions of spinous and granular layer while basal cells showed weaker expression that was confined to cytoplasm. In psoriatic skin syndecan-1 was expressed in the membrane and intercellular junction of cells located in thickened and elongated rete ridges of the epidermis. The strongest reaction was in basal and suprabasal layers and expression diminished through spinous layer. Cells in spinous layer lose syndecan-1 expression, which is opposite pattern to normal skin. Our results suggest that aberrant skin expression of syndecan-1 may be involved in the development of psoriasis.

Keywords Psoriasis - Immunohistochemistry ·

Syndecan-1

Psoriasis is a chronic inflammatory skin disease characterized by exaggerated keratinocyte proliferation. It is estimated that the prevalence of psoriasis varies from 1 to $2 \%$ and increases with age. Current opinion indicates that psoriasis is driven by $\mathrm{T}$ cell-mediated immune responses targeting keratinocytes. However, psoriasis cannot be explained solely on the basis of T-cell activation, and it is likely that an intrinsic alteration in epidermal keratinocytes plays a very important role in disease expression [4].

Syndecans comprise a major family of cell surface heparan sulfate proteoglycans. Syndecans at the cell surface function as coreceptors by binding to ligands and increasing ligand concentration in the pericellular vicinity of their signaling receptors. Several studies indicate their role in adhesion, cell-extracellular matrix interactions, migration, keratinocyte proliferation and differentiation, inflammation, 
and wound healing. In adult tissues, syndecan- 1 is predominantly expressed by epithelial cells and plasma cells, and is expressed to a lesser degree by other cells types (e.g. fibroblasts, endothelial cells) [1].

To determine the expression of syndecan-1 in psoriasis, skin samples from 29 patients (17 women and 12 men, average age 47.3 years) with fully developed psoriasis and skin samples from 14 healthy volunteer persons (eight women and six men, average age 49.8 years) with no personal or family history of psoriasis were immunohistochemically examined using monoclonal antibody against syndecan-1 (CD138, clone MI15, dilution 1:25, DAKO, Denmark). Immunohistochemical analysis was performed following microwave streptavidin immunoperoxidase (MSIP) protocol on DAKO TechMate ${ }^{\mathrm{TM}}$ Horizon automated immunostainer (DAKO). A sample of plasmacytoma that was strongly positive for syndecan- 1 was used as a positive control. The diagnosis of psoriasis was histologically confirmed in all cases and each specimen was re-evaluated by three pathologists (D.T., M.V. and B.K.). None of the patients had received any topical treatment for at least 1 month prior the study. Skin samples of both groups measured between 0.3 and $0.8 \mathrm{~cm}$ (average $0.54 \mathrm{~cm}$ ). The expression of syndecan-1 was analyzed in whole mount section of psoriatic and non-psoriatic skin biopsies under high magnification $(400 \times)$. In addition, the intensity and topography of reaction in the cell, as well as localization of positive cells in the epidermis were evaluated. Statistical analysis was performed using $\chi^{2}$ test. The level of significance was set at $P<0.05$.

Strong syndecan-1 reactivity in epidermal cells in all non-psoriatic and psoriatic samples was observed (Table 1). Statistical analysis showed no significant differences between two analyzed groups $(P>0.05)$. In addition, dermal appendages and some endothelial and inflammatory

Table 1 The intensity of syndecan-1 expression in psoriatic and nonpsoriatic skin

\begin{tabular}{lcc}
\hline & Psoriatic skin $(\%)$ & Non-psoriatic skin $(\%)$ \\
\hline ISI $^{\text {a }}$ & $0(0)$ & $0(0)$ \\
ISI 1 $^{\text {a }}$ & $0(0)$ & $0(0)$ \\
ISI 2 $^{\text {a }}$ & $0(0)$ & $0(0)$ \\
ISI 3 $^{\text {a }}$ & $29(100)$ & $14(100)$ \\
Total & $29(100)$ & $14(100)$ \\
\hline
\end{tabular}

${ }^{a}$ To evaluate the level of syndecan- 1 expression, the percentage of positive cells and the staining intensity were graded on a scale of $0-3$. Staining percentage was labeled as: $0=0 \%$ cells; $1=1-33 \%$ positive cells; $2=34-66 \%$ positive cells; and $3=$ more than $66 \%$ positive cells. Staining intensity was denoted as: $0=$ no staining; $1=$ weak staining; $2=$ moderate staining; $3=$ strong staining. For each sample, the staining percentage and staining intensity scores were multiplied to give staining index. Immunohistochemical staining index (ISI) was labeled as: $0=$ zero; $1-3=$ low $;-6=$ moderate; and $9=$ high cells were also positive. In normal skin syndecan-1 was expressed in full thickness of the epidermis. The strongest reaction was observed in membranes and intercellular junctions of spinous and granular layer while basal cells showed weaker expression that was confined to cytoplasm (Fig. 1a). In psoriatic skin, syndecan-1 was expressed in the membrane and intercellular junction of cells located in thickened and elongated rete ridges of the epidermis (Fig. 1b). The strongest reaction was in basal and suprabasal layers and expression diminished through spinous layer (Fig. 1c). Cells in most upper part of spinous layer showed no expression of syndecan-1 (Fig. 1d).

In our study, syndecan-1 was expressed in all samples of normal skin. The strongest reaction was in suprabasal, differentiating epidermal cells which are consistent with previous investigations $[2,6,8]$. To our knowledge, there are no reports about expression of syndecan-1 in psoriasis. We observed strong syndecan-1 expression in all 29 psoriatic samples, but the pattern of expression was different compared to normal skin. The strongest expression was in proliferating basal and suprabasal layers and expression diminished with cell differentiation. Cells in spinous layer lose syndecan-1 expression, which is of opposite pattern to normal skin.

A role of syndecan-1 in wound healing, epidermal cell proliferation and migration is under extensive research [3, $5,7,9]$. Stepp et al. [9] recently reported that syndecan-1 knockout mice display impaired proliferation and migration of corneal keratinocytes in eye abrasion wound healing. The wounded syndecan-negative corneas failed to initiate proliferative burst $24 \mathrm{~h}$ after wounding. In addition, the migration of corneal cells was hampered by the absence of syndecan-1 [9]. Elenius et al. [5], studied wound healing in mice that overexpress syndecan-1 in several tissues, including skin and blood. The elevated levels of shed syndecan-1 ectodomain in these animals inhibit booth re-epithelization and remodeling of the newly laid matrix in dermis [5]. Ojeh et al. [7] in their investigation, showed role of syndecan-1 in regulation of keratinocytes proliferation during skin development and wound healing. They generated transgenic mice that overexpress syndecan- 1 in the basal layer of the epidermis that harbors epidermal stem cells and transit amplifying cells. Transgenic mice exhibited hyperproliferative skin lesions that were characterized by normal cornified layer, occasional parakeratosis in the granular layer, and increased number of spinous cell layers. The number of suprabasal cell layers was increased and flattening and anucleation of the cells were delayed but terminal differentiation was preserved. The extent of the hyperproliferations varied between different skin locations and between individual mice. Some lesions also contained localized areas of remarkably thickened epidermis that involved some downgrowth into the dermis [7]. Similarities of lesion that exhibited transgenic mice with psoriatic 
Fig. 1 a In non-psoriatic skin syndecan-1 was expressed in full thickness of the epidermis. The strongest reaction was observed in membranes and intercellular junctions of spinous and granular layer. The basal cells in nonpsoriatic skin showed weaker expression of syndecan- 1 that was confined to cytoplasm. b In psoriatic skin syndecan-1 was expressed in the membrane and intercellular junction of cells located in thickened and elongated rete ridges of the epidermis. c The strongest reaction was in basal and suprabasal layers and expression diminished through spinous layer. d Cells in most upper part of spinous layer in psoriatic skin showed no expression of syndecan-1 (the bar in b indicates $200 \mu \mathrm{m}$. a, c and $\mathbf{d}$ are of the same magnification, and bars indicate $50 \mu \mathrm{m}$ )
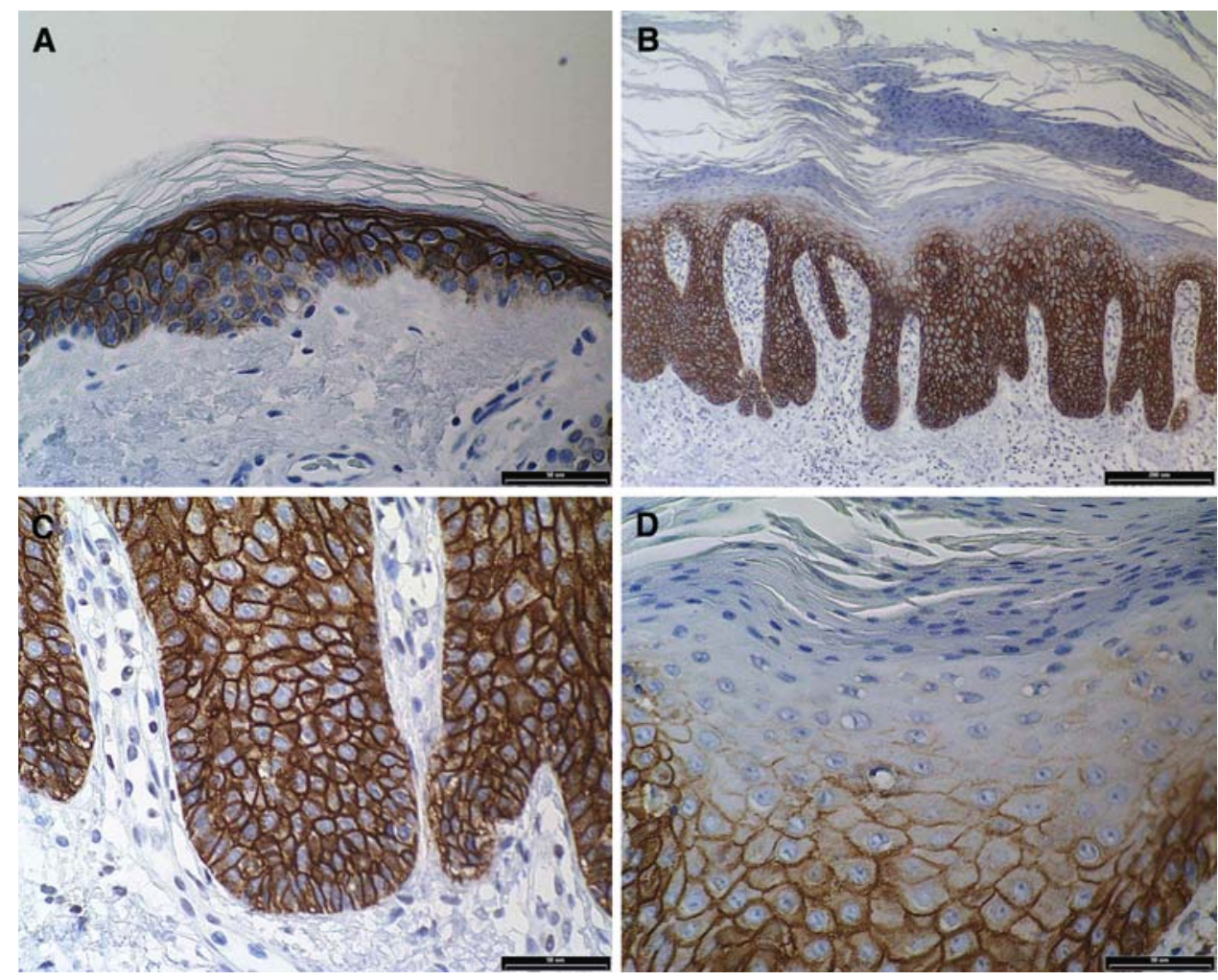

lesion are remarkable. Increased number of suprabasal and spinous cell layers, delayed flattening and anucleation, parakeratosis and remarkably thickened epidermis with downgrowth into the dermis are all histological characteristics of psoriatic skin. In addition, in our investigation, similar expression of syndecan-1 in psoriatic skin and transgenic mice was also observed. The strongest expression of syndecan-1 in psoriasis was in proliferative basal cell layer like in transgenic mice and expression diminished through spinous to cornified layer of the epidermis [7].

Our results suggest that aberrant skin expression of syndecan-1 may be involved in the development of psoriasis. Further studies on larger patient series and in various stages of psoriatic lesions are welcome.

Open Access This article is distributed under the terms of the Creative Commons Attribution Noncommercial License which permits any noncommercial use, distribution, and reproduction in any medium, provided the original author(s) and source are credited.

\section{References}

1. Bartlett AH, Hayashida K, Park PW (2007) Molecular and cellular mechanisms of syndecans in tissue injury and inflammation. Mol Cells 24:153-166
2. Bayer-Garner IB, Smoller BR (2001) The expression of syndecan1 is preferentially reduced compared with that of E-cadherin in acantholytic squamous cell carcinoma. J Cutan Pathol 28:83-89. doi:10.1034/j.1600-0560.2001.280204

3. Bayer-Garner IB, Sanderson RD, Smoller BR (2002) Syndecan-1 is strongly expressed in the anagen hair follicle outer root sheath and in the dermal papilla but expression diminishes with involution of the hair follicle. Am J Dermatopathol 24:484-489

4. de Rie MA, Goedkoop AY, Bos JD (2004) Overview of psoriasis. Dermatol Ther 17:341-349. doi:10.1111/j.1396-0296.2004.04037

5. Elenius V, Gotte M, Reizes O, Elenius K, Bernfield M (2004) Inhibition by the soluble syndecan-1 ectodomains delays wound repair in mice overexpressing syndecan-1. J Biol Chem 279:4192841935. doi:10.1074/jbc.M404506200

6. Inki P, Larjava H, Haapasalmi K, Miettinen HM, Grenman R, Jalkanen M (1994) Expression of syndecan-1 is induced by differentiation and suppressed by malignant transformation of human keratinocytes. Eur J Cell Biol 63:43-51

7. Ojeh N, Hiilesvuo K, Warri A, Salmivirta M, Henttinen T, Maatta A (2008) Ectopic expression of syndecan-1 in basal epidermis affects keratinocyte proliferation and wound re-epithelialization. J Invest Dermatol 128:26-32. doi:10.1038/sj.jid.5700967

8. Sanderson RD, Hinkes MT, Bernfield M (1992) Syndecan-1, a cellsurface proteoglycan, changes in size and abundance when keratinocytes stratify. J Invest Dermatol 99:390-396

9. Stepp MA, Gibson HE, Gala PH, Iglesia DD, Pajoohesh-Ganji A, Pal-Ghosh S, Brown M, Aquino C, Schwartz AM, Goldberger O, Hinkes MT, Bernfield M (2002) Defects in keratinocyte activation during wound healing in the syndecan-1-deficient mouse. J Cell Sci 115:4517-4531. doi: $10.1242 / 10.1242 /$ jcs.00128 